\title{
HARDWARE CONSIDERATIONS FOR HIGH-DYNAMIC-RANGE IMAGING
}

\section{S. BAGRI}

National Radio Astronomy Observatory, Socorro, New Mexico

A. R. THOMPSON

National Radio Astronomy Observatory, Charlottesville, Virginia

\begin{abstract}
The dynamic range of an image obtained using an interferometer array depends on the (interferometer) gain closure errors. This paper discusses hardware imperfections which cause closure errors and how these errors can be minimised.
\end{abstract}

\section{INTRODUCTION}

The dynamic range of an image, defined as the ratio of the maximum brightness to the brightness of the faintest reliable structural feature, often determines the amount of useful scientific data. An image is obtained by Fourier transforming interferometer visibility data at various spatial frequencies. Thus the dynamic range of an image depends on the accuracy of the complex visibility, which is largely determined by the accuracy of calibration of the instrumental gain. The antenna gains are derived from the array equations of the form (e.g. Cornwell 1985):

$$
V_{i j}=G_{i} G_{j}^{*} G_{i j} R_{i j}+e_{i j}+N_{i j}
$$

where $G_{k}$ is the (complex) gain of the $k^{\text {th }}$ antenna, $V_{i j}$ is the measured visibility, $R_{i j}$ is the estimate of the true visibility, $e_{i j}$ is the offset term, and $N_{i j}$ is the system noise. The subscripts $i$ and $j$ refer to antennas $i$ and $j$. Here we are interested in strong sources for which the noise terms $N_{i j}$ can be ignored. The accuracy of the estimated antenna gains is determined by the offset terms $e_{i j}$ and by the deviation from unity of the non-factorable terms $G_{i j}$. Experience shows that with careful design of the hardware it is possible to make the offset terms negligible. The deviations of the non factorable terms $G_{i j}$ from unity are defined as the closure errors; i.e. the closure error for the baseline formed by antennas $i$ and $j$ is given by:

$$
\alpha_{i j}=G_{i j}-1
$$

The dynamic range of a deconvolved image depends on the accuracy with which the sidelobes of the synthesised (dirty) beam are known. The rms error in the sidelobes is proportional to the rms value of the closure errors. Hence, to 
obtain a high dynamic range image, we need to minimize closure errors. This paper is therefore concerned with sources of closure errors and how to minimize them, and is based on experience with the VLA (Napier et al. 1983).

\section{MAJOR SOURCES OF CLOSURE ERRORS IN THE VLA}

A number of instrumental imperfections that result in closure errors were identified during the construction of the VLA (Clark 1978; Thompson 1980; Thompson and D'Addario 1982). Closure errors were calculated using practical instrumental parameters to specify hardware tolerences (Thompson 1980). Major sources of these errors are described below:

(a) Non-identical frequency responses of the signal paths. These include variations in the amplitude and phase across the frequency bands and differences in bandwidths and center frequencies. The effects of differing frequency responses were analyzed in terms of simple models that provide practical criteria for specification of responses. For example, it was found that to keep all closure errors below a level of $1 \%$, amplitude slopes should not exceed $3 \mathrm{~dB}$ across the passbands and sinusoidal ripples should not exceed $2 \mathrm{~dB}$ peak-to-peak. In general, differences in frequency responses produce closure errors predominantly in amplitude, and phase closure errors are small. However, when delay errors are also introduced phase closure errors become important (Thompson and D'Addario 1982).

(b) Delay errors. In normal operation of the VLA the compensating delays that are used to equalize the delays of the signals in traveling through space and through the receiving system are reset to keep the time differences for different antennas within 2 ns. Timing calibration errors and errors in the sampler clock pulses result in overall delay inequalities approaching 3 ns. Test observations have been made in which the compensating delays were carefully set to $1 \mathrm{~ns}$ accuracy. In comparison with normal operation the closure errors were found to be a little smaller, and for any antenna pair they varied less rapidly during the course of an observation.

(c) Phase and amplitude errors in the quadrature networks of the samplers. These networks produce two versions, in phase quadrature, of each baseband signal, which are referred to as the sine and cosine outputs. The networks are required only for operation with the continuum correlator in the VLA. The accuracy of the quadrature phase is about $1^{\circ}$ from $50 \mathrm{MHz}$ down to about $1.5 \mathrm{MHz}$, and errors are significantly greater at lower frequencies.

Early experience with the VLA showed that closure errors were of comparable magnitude in both amplitude and phase and of order $1 \%$ or greater. These errors could not be explained by differences in IF responses or antenna polarizations. Two pieces of evidence pointed towards errors in the quadrature networks of the digital samplers as a major contributor . First, it was found that closure errors increased with decreasing bandwidth. This result is attributable to the fact that the frequencies at the low end of the IF band, where the networks are least accurate, make an increasing relative contribution as the bandwidth is decreased. In contrast, the effects of delay errors, for example, would decrease with decreasing bandwidth. Second, observations using the spectral correlator system, in which only one signal from each of the quadrature networks is used, resulted in lower closure errors. Careful readjustment of the quadrature networks in the early 1980 's reduced 
the continuum closure errors to about $0.7 \% \mathrm{rms}$, but the contribution from the low end of the IF band remains a limitation, especially for the narrower bandwidths.

Evidence that the sources of closure errors are distributed roughly equally between the quadrature networks and other parts of the system is as follows. First, even after readjustment of the quadrature networks, the closure errors measured in the spectral line mode are less by a factor of 2 to 3 when compared with those using the continuum mode correlator. Second, it is possible to obtain two values of both the real and imaginary parts of a visibility measurement using the cosine and sine outputs from the quadrature networks. In normal continuum operation only one value from each of these pairs is retained, since the other values should be the same if there are no errors in the quadrature networks or the samplers. Observations in which both sets of visibility data were recorded and analyzed separately showed only partial correlation between the two sets of closure errors. Furthermore closure errors due to all effects other than the quadrature errors are $\leq 0.1 \%$, as seen while observing in spectral line mode. This indicates that the partial correlation of closure errors between the two sets of data is due to the errors in the quadrature networks. The results are consistent with about half of the closure errors resulting from the quadrature networks.

(d) Non-identical polarization characteristics of antennas. Model calculations for a six-antenna array using circularly polarised feeds, as in the VLA, indicate that an axial ratio of 1.12 for the polarization ellipse produces closure errors of amplitude $0.5 \% \mathrm{rms}$ and $1 \%$ maximum (Thompson 1984). For the VLA feeds the axial ratio is less than 1.07. The mechanical setting error of the feed polarization axes on the sky should not be greater than about $1^{\circ}$. Thus the expected magnitude of the closure errors resulting from differences in the feed characteristics should be much less than $0.5 \%$, if the source is near the center of the beam. Note also that it should be possible to calibrate the cross polarization terms by including them in the solution for the antenna gain, using all polarization products (or even only two parallel-hand terms), but this has yet not been tried in practice.

\section{OTHER SOURCES OF CLOSURE ERRORS}

Some additional non-ideal effects have been considered and are believed to be only minor contributors to closure errors in the VLA with its present receiving system. These are described below:

(a) Linearity of the signal path, spurious signals, and radio interference. Because of limited dynamic range in the RF and IF amplifiers, intermodulation products are formed which result in closure errors. In the VLA, intermodulation products should be at least $20 \mathrm{~dB}$ below the signal levels, and the resulting errors should be $\leq 0.1 \%$. It should be possible to reduce the effects of narrowband spurious signals, both internally generated effects and external interference, by observing in the spectral line mode and deleting channels containing such signals.

(b) Finite image rejection in the conversion of IF signals to baseband. The measured image rejection in the VLA baseband conversion is typically 26-28 $\mathrm{dB}$ after some years of operation, although when the mixers were first constructed the image rejection was more than $30 \mathrm{~dB}$. This results in errors 
in frequency responses, and introduces an error term in the correlator output which depends on the baseband frequency of the image signal and the value of the compensating delay.

(c) Errors in quantizer threshold settings and sampler timing. A discussion of the errors in the quantizer threshold settings and sampler timings is given by D'Addario et al. (1984). The effect of a quantizer DC offset is nullified to first order by the phase switching. The effect of errors in the quantizer threshold settings is corrected by normalizing the correlator output to the geometric mean of the self counts for the corresponding samplers. Second order effects related to DC offsets and errors in quantizer threshold settings also produce closure errors. For low correlation coefficients $(\leq 0.1)$ and threshold changes of up to $5 \%$ closure errors are in the range of 0.05 to $0.1 \%$. For larger correlation coefficients the errors become more important. A discussion of computationally attractive approximations for estimating true correlation coefficients from the measured correlation coefficients, and estimation of quantizer threshold settings using self counts, is given by Schwab (1978). Sampler timing errors are equivalent in their effects to delay errors (D'Addario et al. 1984), and therefore their tolerences should be treated as discussed above.

(d) Cross coupling of noise between antennas (crosstalk) at short spacings. Cross coupling is not believed to be an important factor since closure errors in the $\mathrm{D}$ (close spacing) configuration do not appear to be related to antenna spacing so long as geometrical shadowing of the apertures is avoided.

(e) Antenna pointing errors. Antenna pointing errors should be included within the antenna-related gain terms, so long as the source is small compared with the antenna beam, and the pointing errors are not so large that they result in polarization mismatch. If the source size is a significant fraction of the beamwidth then the results cannot be represented by a single gain factor because the polarization errors vary over the beam. As Clark (1981) has pointed out, if the source is dominated by a strong component this should be placed at the center of the field.

(f) Atmospheric effects. The effects of atmospheric propagation are included within the antenna gain terms so long as they do not vary greatly on the time scale of the integration of the visibility. Long integration periods for visibility during poor weather conditions increase closure errors because of the decrease in the coherence.

Closure errors in the VLA resulting from each of the above effects are estimated to be at the level of $0.1 \%$ or less. None of them has been identified as a limiting factor in the dynamic range obtainable at this time. However, eventhough each such effect may make only a small contribution, the combination of several of them could be important.

\section{CALIBRATION OF CLOSURE ERRORS}

If closure errors are sufficiently stable they can be removed by calibration. In this approach values of closure errors, $\alpha_{i j}$, are determined, and used in deriving the visibility data using eqs. (1) and (2). Test observations with the VLA, made in the late 1980 's, indicate that under reasonable conditions closure errors are stable to $0.1 \%$. 
We now consider the dependence of closure errors upon the instrumental phase. Let the arguments of the signals before the quadrature networks for antennas $\mathrm{i}$ and $\mathbf{j}$ be $\theta_{i}$ and $\theta_{j}$. Let the errors introduced in the sine and cosine outputs of the quadrature networks be $\Delta_{s i}$ and $\Delta_{c i}$ for antenna $i$, and $\Delta_{s j}$ and $\Delta_{c j}$ for antenna $j$. The real part of the correlator output for a signal component at intermediate frequency $\nu$ for the baseline formed by antennas $i$ and $j$ may be written as:

$$
R e\left[V_{i j}\right]=<\cos \left(2 \pi \nu t+\theta_{i}+\Delta_{c i}\right) \cos \left(2 \pi \nu t+\theta_{j}+\Delta_{c j}\right)>
$$

This may be simplified as:

$$
R e\left[V_{i j}\right] \approx\left[1-\frac{\left(\Delta_{c i}-\Delta_{c j}\right)^{2}}{2}\right] \cos \Theta_{i j}-\left(\Delta_{c i}-\Delta_{c j}\right) \sin \Theta_{i j}
$$

where $\Theta_{i j}=\theta_{i}-\theta_{j}$, the angular brackets indicate a time average, and for simplicity we have considered signals of unit amplitude. For small values of the $\Delta$ terms eq. (3) becomes

$$
R e\left[V_{i j}\right] \approx \cos \Theta_{i j}-\left(\Delta_{c i}-\Delta_{c j}\right) \sin \Theta_{i j}
$$

Similarly, for the imaginary part we have:

$$
\operatorname{Im}\left[V_{i j}\right] \approx \sin \Theta_{i j}-\left(\Delta_{c i}-\Delta_{s j}\right) \cos \Theta_{i j}
$$

The second term on the right-hand side in eqs. (4) and (5) results from the imperfections in the quadrature networks, and contributes to the closure errors. These terms are functions of $\Theta_{i j}$, which includes atmospheric and instrumental phase effects that vary on time scales of minutes to hours. Thus the visibility errors should be more stable when the array is operated in the real-time phased mode (i.e. $\Theta_{i j}=0$ ). An extension of the analysis in eqs. (4) and (5) to include amplitude and phase errors in the quadrature networks leads to more complicated expressions, which again contain error terms that are functions of $\Theta_{i j}$. Thus again we expect the stability of the closure errors to be increased by phased-array operation. Note, however, that real-time phasing is only practical in cases where the field is dominated by a point source. Observations of strong calibration sources (e.g. 3C84), both with and without real-time phasing of the array, confirm that phasing reduces the variability of closure errors.

With the continuum mode correlator the rms closure errors are greater by a factor of 2 to 3 than with the spectral correlator. Operating the array with real-time phasing in the continuum mode increases the stability of the closure errors, and hence also the effectiveness with which they can be removed by calibration (see, for example, Walker et al. 1988). Note that if the form of the dependence of the closure errors on the phase is known, as in the case of the phase errors considered in eqs. (4) and (5), then it should be sufficient to calibrate the instrumental factors in the expressions for the closure errors using values of the phase for each baseline that are obtained from determination of antenna gains. Investigation of this possibility has not been pursued.

When observing 3C84 with the spectral correlator, it has been found that the stability of the closure errors is further improved by about a factor of two by real time phasing the array. This may be due to inadequate correction for 
quantization effects, resulting in errors that change with the measured visibility phase.

\section{CONCLUSIONS FROM EXPERIENCE WITH THE VLA}

With regard to system design and operation, the principal points obtained from the experience with the VLA can be summarized as follows:

(a) Observing in spectral line mode to avoids errors contributed by the quadrature networks. Closure errors are a factor of 2 to 3 smaller in spectral line mode than in the continuum mode. However, observing in spectral line mode reduces the signal to noise ratio because the maximum usable bandwidth is reduced.

(b) The system delays should be calibrated and set as accurately as possible. Not only are closure errors thereby reduced, but they are more stable and thus easier to calibrate. Carefully setting the instrumental delays prior to the observations enabled Hines et al. (1989) to obtain a dynamic range of 150,000 on $\mathrm{M} 87$ in the A configuration at $6 \mathrm{~cm}$.

(c) With the spectral correlator, the limitations on the dynamic range are believed to result from a combination of effects which may include polarization mismatch of the antennas, finite image rejection, and timing or level errors in the samplers. Closure errors from these effects in combination are of order 0.1$0.2 \%$, and it is not known which are the most important.

(d) Calibration of closure errors as characteristics of the various antenna pairs has proved to be effective. This is likely to be a more practical way to reduce closure errors than an attempt to eliminate small departures from ideal behavior that may be widely distributed within the system. The level to which errors can be reduced will depend upon the stability of the electrical characteristics of the overall system.

(e) Dynamic ranges of up to 200,000 have been achieved with the VLA in the A (longest spacing) configuration. For example, in continuum mode, dynamic ranges of 145,000 on 3C84 (Bagri 1990) and 119,000 on 3C120 (Walker et al. 1988) have been achieved. In spectral line mode Perley (private communication) has obtained a dynamic range of 190,000 on 3C84. Several attempts to improve the performance have been unsuccessful for reasons that are not fully understood.

\section{6. $\underline{\text { VLBI }}$}

The longer baselines and consequently greater natural fringe frequencies that occur in VLBI should aid in discriminating against unwanted responses such as interference, and hence in obtaining greater dynamic range. In VLBI, spectral type correlators are generally used to allow offsets in timing and fringe frequency to be accommodated, and bandpass calibration is therefore usually possible. However dynamic range obtained in VLBI imaging has generally been one order of magnitude less than has been obtained using connected element arrays, possibly because of the diversity of antennas and receivers often combined in VLBI arrays. The VLBA will be the first VLBI imaging array with closely matched receiving systems at the various antennas. Also, as discussed in section 2, delay errors can result in serious closure errors in 
amplitude and phase of the visibility. The pulse calibration system planned for the VLBA (see Thompson and Bagri, this volume) should help in calibration of such instrumental errors. Closure errors $\leq 0.1 \%$ is a goal for the VLBA.

\section{NEW REQUIREMENTS AND TECHNICAL POSSIBILITIES}

An important requirement in future arrays will be increasing the bandwidth to provide greater sensitivity, especially at wavelengths shorter than a few centimeters. Spectral correlators of the FX type will allow bandpass calibration, so frequency responses of the hardware need not be a performance limitation. Optical fiber links are likely to be widely used in connected-element systems, and their wide bandwidths provide the possibility of transmission of the signals from the antennas to the correlator in digital form.

Digital transmission was considered during the design of the VLA receiving system, and rejected for the following reasons. First, with two bits per sample the bit rate would be four times the analog signal bandwidth, and it would not have been easily accommodated within the waveguide bandwidth. Second, it appeared desirable to transmit the signals in analog form so as to be able to perform the final (baseband) filtering at the central electronics building. There are substantial reasons for performing the frequency conversion to baseband and the baseband filtering at one location. These are, first, that fine tunability is required in the final frequency conversion to allow selection of spectral lines, and generating the necessary phase-stable oscillator signals separately at each antenna would substantially complicate the local oscillator system. Second, matching of the frequency responses is most accurately maintained if all of the final filters are located in the same environment.

In the more recently designed Australia Telescope the signals are sampled at the antennas and transmitted digitally via optical fibers to the central location. There they are reconstituted in analog form, filtered for narrow band operation as required, and then sampled again before going to the digital correlator. This scheme has the advantages of non-distorting digital transmission, while keeping the final frequency converters and filters in a single location. Since the signals are sampled twice the loss of sensitivity resulting from quantization occurs twice, but the loss at the first quantization is low since four bits per sample are used.

Digitizing the signals at the antennas eliminates bandpass variations due to reflections in long transmission lines and variations in frequency responses of components required in extensive analog signal processing. Further, the use of FX correlators allows easy bandpass calibration. Such techniques hold promise of continuing improvements in the dynamic range of future systems.

\section{REFERENCES}

Bagri, D. S. 1990, VLA Electronics Memo. No. 216, NRAO, Socorro, NM. Clark, B. G. 1978, VLA Electronics Memo. No. 171, NRAO, Socorro, NM. Clark, B. G. 1981, VLA Scientific Memo. No. 137, NRAO, Socorro, NM. Cornwell, T. 1985, in Synthesis Imaging, Workshop held Aug. 5-9, 1985, Socorro, NM. , eds. R. A. Perley, F. R. Schwab and A. H. Bridle, p. 137. D'Addario, L. R., et al. 1984, Radio Science, $19,931$. 
Hines, D. C., et al. 1989, Ap. J., 347, 713.

Napier, P. J., et al. 1983, Proc. IEEE, 71, 1295.

Schwab, F. 1978, VLA Computer Memo. No. 150, NRAO, Socorro, NM.

Thompson, A. R. 1980, VLA Electronics Memo. No. 192, NRAO, Socorro, $\mathrm{NM}$.

Thompson, A. R. and D'Addario, L. R. 1982, Radio Science, 17, 357.

Thompson, A. R. 1984, VLB Array Memo No. 346, NRAO, Socorro, NM.

Walker, R. C., et al. 1988, Ap. J., $\underline{335}, 668$. 